\title{
Peculiarities of publication activity in humanities and social sciences: analysis and prospects
}

\author{
Tatyana Sidorenko ${ }^{1, a}$, Irina Kuznetsova ${ }^{1}$ \\ ${ }^{1}$ Tomsk Polytechnic University, Institute of Cybernetics, 634034 Tomsk, Russia
}

\begin{abstract}
The goal of the paper is to analyze the publication activity as a phenomenon, which has taken the firm position in the academic environment as a criterion of assessing the professional efficiency of the university academic staff. The authors consider the peculiarities of humanities and social sciences in comparison with technical and natural sciences from the point of initial possibilities to increase the rate of publication activity and a citation index. In order to have a clear insight of the humanities and social fields, we needed to identify the subject and the object they research and the outcomes they are able to represent as scientific results. The data showed a certain inequality at the start. Social sciences have poor pragmatic influence. They are characterized by targeted and valuable measurements but not by pragmatic parameters. As the main instruments to solve or balance this issue, we discuss the potential value of the following motivation factors as money, career perspectives, and social safeguards, which are able to impact the situation better.
\end{abstract}

\section{Background history}

In 2013, National Research Tomsk Polytechnic University joined the TOP-15 leading universities of Russia in the framework of the development program of leading research universities. These are the universities that, upon competitive selection, were chosen by the RF Ministry of Education and Science to make the TOP-100 positions in the world university ranking under the Decree of the President and within the shortest time possible, i.e. 7 years in this case. In this respect, TPU has designed the university development program and the roadmap defining its priority development fields following the criteria of the world ranking agencies, among which there is the Academic Ranking of World Universities (ARWU), (published since 2003); QS World University Rankings or QS (published since 2000); Times Higher Education World University Ranking (THE) or Times (published since 2010). Such rankings seek the following goals: 1) evaluation of higher education at all levels (education, science, management, financing, and infrastructure); 2) providing consumers with reliable information about educational services.

Most Russian universities are aimed at the QS ranking. The principal QS criteria include:

1) academic reputation $(40 \%)$;

2) a number of visiting specialists (5\%);

3) employers' qualitative evaluation of graduates' training $(10 \%)$;
4) a number of international students who study within of academic mobility programs $(5 \%)$;

5) a student-faculty ratio (20\%);

6) a citation index (20\%) [1].

Therefore, the university revised its strategic objectives focusing more on TPU development as a research university and becoming one of the leaders in the field of resource-efficient technologies, thus solving the global tasks of the mankind in its strive for sustainable development. The objective is decomposed into a number of key areas:

- world-class research in the field of resourceefficient technologies;

- $\quad$ globally competitive engineering education;

- replication of the best academic and engineering practices;

- strategic partnership with academic and business community;

- training and involvement of talented students, scientists and faculty;

- social importance and responsibility from a regional, national and global perspective [2].

The university development program implies the achievement of key indicators in certain aspects. Let us take a closer look only at those that are relevant to research and academic staff publication activity, which is, on average, comprised of the following:

- a number of papers per one research and academic staff in Web of Science (WOS) and Scopus databases,

\footnotetext{
${ }^{\mathrm{a}}$ Corresponding author: sidorenkot@tpu.mail.ru
} 
excluding their duplication: $2013-0.6 ; 2014-0.8 ; 2015$ - $1.1 ; 2016$ - 1.5; 2017 - 2.2; 2018 - 2.9; 2019 - 3.6; $2020-4.7$;

- an average citation index per one research and an academic staff member calculated per totality of papers in WOS and Scopus databases, excluding their duplication: $2013-2.4 ; 2014-2.9 ; 2015-3.6 ; 2016-$ $4.6 ; 2017$ - 5.7; 2018 - 7.0; 2019 - 8.9; $2020-11.1$.

Thus, figures show that on average one research and academic staff member shall foster the 7.8-fold improvement of its publication activity within 7 years, while the average number of citations per one research and academic staff member shall be increased 4.6 times as much.

It appears that the scientific performance of an individual faculty member and a researcher within the system of university overall performance shall be measured by various criteria, namely the citation index, which is often called the scientometric indicator of publication activity, the number of papers and the impact factor. The latter one being a measure reflecting the average number of citations to recent papers published in a journal registered within the WOS (in any given year out of the last five years starting from the year preceding the current one) is multiplied by the impact factor of this journal in a corresponding year.

\section{Scientific citation indices}

The origin of scientific citation indices dates back to the $1870 \mathrm{~s}$ of the $19^{\text {th }}$ century almost alongside with the appearance of legal citation indices, known as the Sheperd's citations (1873) and a comprehensive index of scientific papers focusing on medical sciences, the Index Medicus (1879). In the Unites States of America the first unified quantitative indicators related to evaluation of research were published in 1972 in the report called the Science Indicators.

In 1987, China also joined the project of the national science citation index (Chinese Science Citation Index). However, it was not the only project in China, since in 1988 there appeared another alternative (a competitive index called China Scientific and Technical Papers and Citations). In 1995, Japan enters upon the creation of its national citation index (Citation Database for Japanese Papers).

In Russia, the notions of 'publication activity' and 'scientific citation index' have only started being actively used since autumn of 2009 as it was then when the RF Ministry of Education and Science approved the decree on the use of standard methodology for performance evaluation of scientific organizations engaged in research and technology.

\subsection{Issues of citation indices in social sciences and humanities}

Notwithstanding the fact that the citation index is a mature phenomenon being used for a relatively long time for scientific performance evaluation there is a fair bit of debates among scientific community representatives over its reliability and, above all, its universality. In other words, there is a problem of some initial scientific inequality arising from such notions as priority and secondary scientific areas, globally and locally important research, all being fundamental for citation frequency.

The second problem is that of quantity dominating over quality, which is caused by high competition that occurs in recent years within the academic community, as well as drive for commercialization of "the economic sector" as scientific publications. As McGarty aptly summarizes: It is not just a bad measure, it is an invitation to do bad science [3].

The third problem is not a consequence of peculiarities of social sciences and humanities and refers to the notion of the researcher's impact factor. It is attributed to the journal impact citation window, which is calculated as the average number of references to each journal in a current year with respect to 'citable items' published in that journal during the two or five preceding years [4].

However, there is not an optimal fixed impact maturity time valid for all the fields. In some of them two years provide a good performance, whereas in others three or more years are necessary. Therefore, there is a problem when comparing a journal from a field where the impact matures slowly with a journal from a field, in which the impact matures rapidly.

For example, in biomedical fields long reference lists with more than fifty items are common, but in mathematics short lists with fewer than twenty references are the standard [5]. These differences are a consequence of citation cultures, and can lead to significant differences in the journal impact factor across fields of science since the probability of being cited is affected. In this sense, this is the factor that the most frequently has been used in literature to justify the differences between fields of science, as well as the most employed in source-normalization $[6,7,8]$.

As an example, a perusal of the last issue of 2007 of the Journal of International Business Studies shows that even in this most optimistic case (i.e. the final issue of 2007) very few references to publications in 2005 and 2006 are found in the ten papers published in this issue. Out of more than 700 references in this issue, only 20 referred to publications in 2005 and a mere 7 to publications in 2006 (i.e. less than $4 \%$ of the total number of citations) [8].

The sub-problem flowing from this is that one third of these citations were self-citations. This is not entirely surprising given that of the ten papers in this issue, six were submitted before 2005 (four in 2004, one in 2003, and one in 2002). Of the remaining four, two were submitted in January and February 2005 and hence cannot realistically be expected to include references to 2005 papers. The final two papers were submitted in January and May 2006 [7].

However, let us focus on some 'inequality' of scientific fields. It is obvious that research in the field of natural and technical sciences will differ considerably from that of social sciences and humanities. For instance, biochemistry and molecular biology are disciplines that are characterized by a high number of citations and short 
publication lags. Hence, the use of a 2-year citation window might have been justified. However, this is not true for the majority of other disciplines, where knowledge takes much longer to be disseminated. Although Thomson ISI has recently introduced a 5-year impact factor, a 2-year impact factor is still the most commonly used [7].

This does not provoke any doubt in terms of the fact that publication activity of research and academic staff working in the field of social sciences and humanities is a priori much lower than those of natural and technical sciences. Moreover, this fact is not random but rather systemic.

\subsubsection{Influence of science field peculiarities on the impact factor and citation index}

It is above all necessary to characterize knowledge peculiarities of social sciences and humanities, i.e. to identify peculiarities of its scientific organization. This will make a scientific model of social sciences and humanities more accurate and hence, will allow more distinctly presenting peculiarities of scientific publication activity of authors working in this field.

Objects of social sciences and humanities are so diverse that it is impossible to give their comprehensive overview though a single approach or a paradigm. An object of perception within social sciences and humanities does not align with itself, it is rather present in the process of self-evolution, and hence, its classical perception is not only complicated but is meaningless.

Let us consider the model of social and humanitarian knowledge, ways of its organization and peculiarities as well. Ideas justifying the fact that rationality is not the only principle defining human mindset and behavior form the basis for social and humanitarian knowledge. Will, feelings and emotions are equally important here. This means that an individual does not perceive herself/himself as a fully known reality. Moreover, at any time an individual acts as an unknown human being thus creating an interest towards her/him and her/his internal world. It is that interest that serves a key imperative of social sciences and humanities.

With regard to the study of publication activity phenomenon in social sciences and humanities the revealed features justify the following. The number of adherent points in dividing scientific areas is minimum similar to the number of citations. Especially this concerns authors coming from different cultures, civilizations and eras. Even one and the same topic viewed by representatives of different countries may be perceived and thus presented in an absolutely different reality, conditions, environment, etc.

Topics of local history aiming at designing the local historical memory and description of history of a certain place - a locus may serve as examples of this kind. We cannot deny the fact that such areas have their own, although not too dynamic, development in the world. Thus, in Great Britain in the second half of the $20^{\text {th }}$ century there appeared centers of scientific study of local history (including the British Association for Local History) and the following journals were issued: The Local Historian, Local History News, etc. In turn, the Modern Local History Center, an interuniversity scientific and educational institution, has been functioning in Russia on the basis of North-Caucasus Federal University [9].

Peculiarities of social and humanitarian aspects of research also complicate the development of crosscultural and international relations (what is interesting to one culture or country may lack interest for another). So, such areas as translation studies, study of the national language and culture, law and less often pedagogy and sociology are exposed to some isolation in terms of knowledge dissemination.

On the one hand, dissociation of monitoring systems during knowledge exchange makes it possible to expand personal worldview boundaries and adopt successful practices. But on the other hand, the environment where any given practices and methods are implemented often prevents their implementation globally. All this combined cannot but affect the citation frequency thus creating new barriers for potential scientific cooperation.

Social sciences and humanities have poor pragmatic influence. They are characterized by targeted and valuable measurements but not by pragmatic parameters. Obviously, if compared to natural and technical sciences, the concept of novelty during the study within the field of social sciences and humanities does not bear exactly the same value. Here the focus is more on the function of cultural integrity in the form of valuable, standard, regulating and other aspects of modern dynamics. Unfortunately, this function in particular is not strongly sought by a society, business and a state, and, therefore, gets the minimum financial support.

Search for new knowledge is present but more often it is not the primary focus. Therefore, authors of publications in the field of social sciences and humanities are more focused on demonstration of the variety of reality rather than on search of what is yet unknown to the mankind.

\subsubsection{Examples in numbers}

It is becoming apparent that publication activity of representatives of social sciences and humanities will a priori be lower and, hence considerably lower.

Let us give an example with numbers. If we follow the official reports on the overall university performance, then, in 2015 , the publication activity of the Institute of Humanities and Social Sciences made 0.015 papers per one research and academic staff member whereas the alluniversity indicator equals 0.63 papers. Thus, lag of research and academic staff of this institute against the university in general makes over a 40 times difference. The given facts should be taken into account when developing further actions and measures aimed at the improvement of publication activity and during the 
comparative study of the overall performance of the university divisions.

It is worth mentioning that publication activity on a scale necessary for leading higher education institutions is not really dependent on a scientific field. According to statistics, in Russia, during 2011-2012, the total cohort of researchers, post-graduate and doctoral students, as well as the faculty of higher education institutions amounted to 883.832 persons. These data prove that only every seventh Russian researcher is published in WOS journals [10].

Any scientific publication is only the "top of an iceberg", they are not taken "from nowhere". For any paper to be published there should be a relevant study of the matter, its financing, and authors' participation in corresponding scientific events, etc. It is important that employees are motivated for research, and this will take some time. Provided all these combined a publication activity of representatives of social sciences and humanities may be improved.

Another pitfall, typical for Russian institutions and academic staff, is a low language proficiency, which is conditioned with different historical and linguistics factors. It is just so happens that all WOS and Scopus journals use English as the language of publications. A proportion of Russian and English journals available in data base, which are used to calculate the citation index is 1 to 46 . In this regard, according to the latest statistic data, the citation index of Russian scientists in general by 2013 in the international data base was only $1 \%$ [11].

Translation of publications into English does not save the situation because it requires time. But time in this case values more than gold. Thus, a paper is ready, then we spend time to translate it, the translated paper is to be reviewed and then published. After publication it should take up to six months before the paper reaches scientific community and finds like-minded people who are supposed to ensure the citation index to the author. Under the most optimistic projection, the entire process takes no less than one year. Considering the fact of unpopularity and inertness of social sciences and humanities it will take up even more.

\section{Measures to address the situation}

What measures can be taken within the higher education institution in order to improve the situation? As a rule, money is always a good incentive. However, a person can only expect up to $18-20 \%$ increase in publication activity in WOS annually, but only within the first three-four years with further decrease normally the resources are quickly exhausted as they do not imply the reproduction of new published authors. At the same time expenses for science are reduced and hence, during the last five years made: 2012 - $323.3 \mathrm{bln}$, $2013-327.7$ bln, 2014 - 283.6 bln rubles, which is about $0.7 \%$ GDP.

This shows that financial support is not enough to solve the issue. Even if to increase a single payment for a paper, it will unlikely lead to a quantum leap in publication activity since the cohort of potential authors is limited and their writing opportunities are not infinite. Therefore, there is a need to involve other motivating factors, such as career ambitions, privileges and guarantees or safeguards. Personal motivation being a key one in scientific activity in relation to other aspects of interest was several times proved by management practices. In general, motivated employees have higher motivation in relation to their intellectual success.

The following can be considered as career ambitions: promotion, additional duties, involvement in project works, participation in significant academic events, internships. The same "nominations" serve as privileges and safeguards, alongside with factors of psychological and emotional environment, responsibility for safety, reliability, protection, satisfaction, demand that can be added to the above list. The correction factor used in evaluation of scientific activity of employees within social sciences and humanities, which to some extent correlated a ratio of indexes in technical fields and humanities, may also serve as another privilege.

Correction factors will help to avoid judgmental evaluations negatively impacting the motivation of a person and will diminish the use of such terms as "effective" and "inefficient researcher", which are widely used recently.

\section{Conclusions}

In the conclusion it should be noted that similar to qualitative indicators with enough reasons for speculation the quantitative indicators of scientific activity evaluation have many disadvantages. The main task here is to try to compensate the redundancy or lack of some indicators with others. On the one hand, there are many circumstances influencing a scientist's citation index. On the other hand, it is the indicator which certainly correlates with the level and efficiency of the scientist. At the same time, its calculation in fields related to social sciences and humanities shall not be exclusively made in numbers; it shall also imply a more complex evaluation taking into account initially unequal positions, low ranking of humanities and an extra cultural and educational task, which is assigned to representatives of social sciences and humanities of any higher education institution.

\section{References}

1. T. Sidorenko, T. Gorbatova, Procedia-Social Behavioral Sciences, 166, 464 - 467 (2015)

2. Competitiveness enhancement program work plan (work flow chart) of National Research Tomsk Polytechnic University for 2013-2020. Tomsk. (2013) Available at: http://tpu.ru/today/programs/viu/

3. C. McGarty, Collective guilt in Australia. Paper presented at the expert meeting on Collective Guilt (Amsterdam, 2000)

4. E. Garfield, Science, 178(4060), 471-479 (1972)

5. P. Dorta-Gonzalez, M.I. Dorta-Gonzalez, Comparing journals from different fields of science 
and social science through a JCR subject categories normalized impact factor. Scientometrics (2013)

6. L. Leydesdorff, Bornmann, Journal of Informentrics, 5(1), 87-100 (2011)

7. H. F. Moed, Journal of Informetrics, 4(3), 265-277. (2010)

8. M. Zitt, H. Small, Journal of the American Society for Information Science and technology, 59(11), 1856-1860 (2008)

9. Theoretical dictionary. Theory and methodology of historical science (2014)

10. V.V. Pislyakov, Sociological journal, 1, 128-140 (2007)

11. N. Titova, Development strategies of Russian Universities: meeting new challenges (Moscow. MAKS press, 2008) 